\section{Amorphous Metals as New Materials for Transducer Applications}

\author{
C. Lindner, A. Dommann, G. Staufert, M.-A. Nicolet* \\ Institut für Mikrosystemtechnik, Neu-Technikum Buchs, CH-9471 Buchs, Switzerland \\ *California Institute of Technology, Pasadena, CA 91125
}

\section{Introduction}

Appropriate physical and chemical characteristics as well as reproducibility and long-term stability of new materials are sustantial for their use in microsystems. Amorphous TaSiN thin films have proven to successfully meet the requirements as diffusion barriers for metal/ silicon contacts in micro-electronics [1]. They are thermodynamically stable with their adjacent materials $(\mathrm{Al}, \mathrm{Cu}, \mathrm{Si}$ or silicide) and, due to their lack of grain boundaries, show a satisfactory opaqueness for metals. Moreover, the resistivity of these TaSiN films is a few hundred $\mu \Omega \mathrm{cm}$ for nitrogen concentrations less than $50 \%$, representing adequate electrical conductivity [1]. In terms of mechanical properties, a Young's modulus of $190 \mathrm{GPa}$ has been measured for the TaSiN films [2] which is of the order of that of silicon. Furthermore, compressive stress in the $100 \mathrm{MPa}$ range of the asdeposited films could be reduced by annealing: heating up to $450^{\circ} \mathrm{C}$ resulted in a monotonic stress decrease and subsequent recooling even reversed into the tensile direction [2]. Finally, the amorphous microstructure of this new material may impede fatigue and wear effects, as often observed in metals. Considering all these outstanding material properties, amorphous TaSiN films are promising candidates for application in micro-electromechanical devices. By adding $\mathrm{Al}$ as a sacrificial layer, the fabrication of freestanding TaSiN surface microstructures was achieved.

\section{Sputter Deposition and Film Pro- perties}

The amorphous TaSiN films were deposited by reactive sputtering in an $\mathrm{Ar} / \mathrm{N}_{2}$ plasma with a total gas pressure of $10^{-2}$ mbar. $\mathrm{A} \mathrm{Ta}_{5} \mathrm{Si}_{3}$ target was employed and sputtering was performed by applying a DC power of $800 \mathrm{~W}$. Compared with the RF mode, the DC sputtering resulted in a higher deposition rate of about 10 $\mathrm{nm} / \mathrm{min} \cdot \mathrm{kW}$.

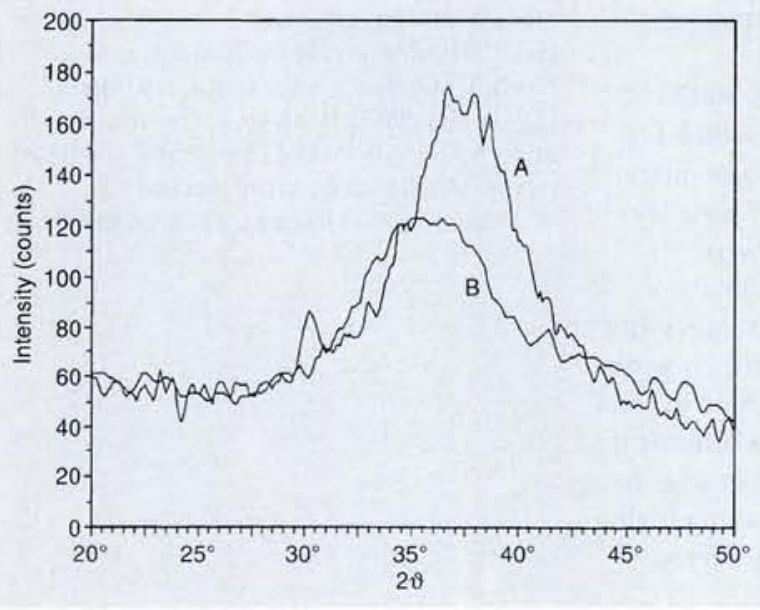

Fig.1. X-ray diffraction pattern of TaSiN films sputter-deposited at different partial gas pressure ratios of $\mathrm{N}_{2}$ to $\mathrm{Ar}: \mathrm{A}-5 \% \mathrm{~N}_{2} ; \mathrm{B}-10 \% \mathrm{~N}_{2}$
No extern heating was applied. The substrates were exposed to the plasma induced heating at around $100^{\circ} \mathrm{C}$. An in situ pre-etch in an $\mathrm{Ar} / \mathrm{O}_{2}$ plasma significantly improved the adhesion of the films.

In our investigation we also applied a new physical measurement system for micro-electromechanical devices, called the X-ray rocking curve method, which allows the strain in a crystal to be measured. With high-resolution instruments (high-resolution X-ray diffractometer, HRXRD) a strain tensor profile can be rec-orded and thus a detailed surface strain analysis is possible [3]. Strain on the wafer induced by amorphous TaSiN layers can be documented. HRXRD is a non-destructive method with a high strain sensitivity. By using Hooke's law, the stress can be determined from the lattice strain. HRXRD measurements were performed to investigate the substrate strain induced by the metal layer using the very intense beam of a MPD $1880 / \mathrm{HR}$ Philips diffractometer with a Bartels monochromator. We have a powerful method for the investigation of thin films up to the order of some $\mu \mathrm{m}$, the typical range of micromachined actuators.

Crystallinity analysis was carried out using a Philips PW1050 computercontrolled X-ray diffractometer with filtered $\mathrm{CuK} \alpha$ radiation. X-ray diffraction patterns of the TaSiN films deposited at different $\mathrm{N}_{2}$ partial pressures are illustrated in Fig. 1. Pattern A is obtained from a sample with $5 \% \mathrm{~N}_{2}$ and pattern $B$ from a sample with $10 \% \mathrm{~N}_{2}$, where the percentage refers to the partial gas pressure ratio of $\mathrm{N}_{2}$ to $\mathrm{Ar}$ in the sputtering process. To obtain a well-defined background signal, all measurements were performed on TaSiN films deposited on [100] Si wafers. It is clearly seen that both samples are X-ray amorphous, although the maximum is shifted, between $5 \%$ and $10 \% \mathrm{~N}_{2}$. Cross-sectional TEM measurements revealed [2] that this is also the range where the change from amorphous to crystalline occurs. RBS measurements of these TaSiN films yielded the following atomic compositions (with an error of about $10 \%$ ) of $\mathrm{Ta}_{49} \mathrm{Si1}_{4} \mathrm{~N}_{37}$ for $5 \% \mathrm{~N}_{2}$ and $\mathrm{Ta}_{44} \mathrm{Si}_{14} \mathrm{~N}_{42}$ for $10 \% \mathrm{~N}_{2}$. The crystallisation temperature of the $\mathrm{TaSiN}$ films as determined by $\mathrm{X}$-ray diffraction was about $800{ }^{\circ} \mathrm{C}$ (about an hour), representing a high thermal stability.

A contact AFM picture of a sputtered TaSiN surface is shown in Fig.2; it was taken by an AutoProbe LS Scanning Probe 


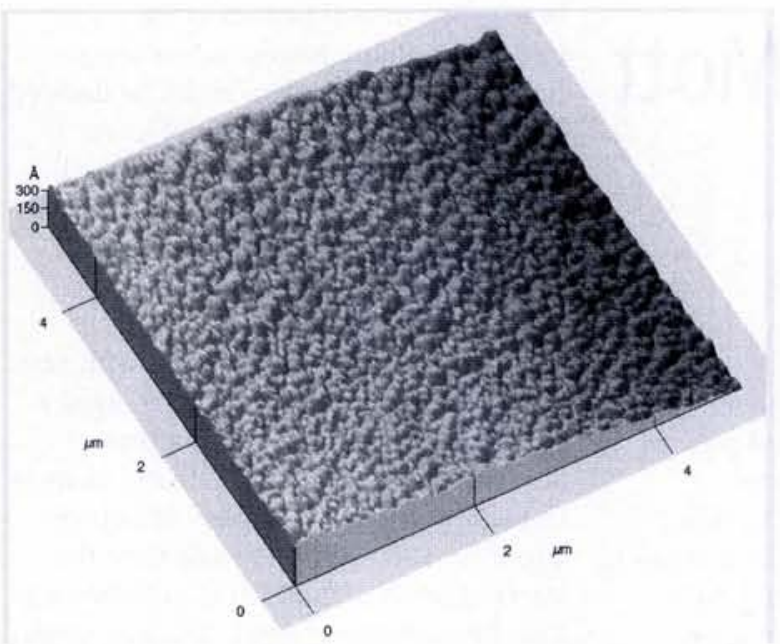

Fig. 2. Contact AFM image of a section of sputtered TaSiN surface

Microscope by Park Scientific Instruments with an $0.6 \mu \mathrm{m}$ microlever.

The surface is extremely smooth, having a roughness of less than $20 \mathrm{~nm}$. Thus, amorphous TaSiN films are promising candidates for micromechanics applications requiring low friction. Preliminary friction measurements between two coated samples revealed smaller values than in the case of steel.

\section{Surface-Micromachined TaSiN Structures \\ Sacrificial layer technology has} become a well-established surface micromachining technique for the fabrication of three-dimensional microstructures from thin films on any process-compatible substrate. An all-metal combination has been chosen for the fabrication of free-standing amorphous TaSiN films (1-2 $\mu$ m thick) with $\mathrm{Al}(2-4 \mu \mathrm{m})$ as a suitable sacrificial layer. In particular, both layers can be sputter deposited in a low temperature step (less than $300^{\circ} \mathrm{C}$ ), offering the possibility for post-processing on a substrate with prefabricated electronic components. The patterning of the TaSiN was performed in a $\mathrm{SF}_{6} / \mathrm{O}_{2}$ plasma using a photoresist mask; further fluorine-based dry etching processes are described in [4], for example. The lateral etching of the sacrificial $\mathrm{Al}$ in a standard $\mathrm{H}_{3} \mathrm{PO}_{4} / \mathrm{NHO}_{3}$ mixture was highly selective, i.e. the etchant did not attack the TaSiN film.

A fabricated TaSiN microbridge is shown in Fig. 3. The slight buckling of the free-standing double-clamped beam indicates that the compressive stress of the TaSiN film was in agreement with the measurements by Reid [2]. This compressive stress in the $100 \mathrm{MPa}$ range was reduced by heating up to $450^{\circ} \mathrm{C}$.

Potential applications for such amorphous metal microbridges are

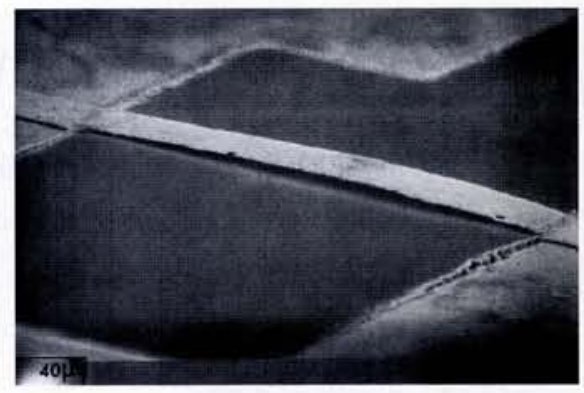

Fig.3. SEM of a free-standing amorphous TaSiN microbridge with $\mathrm{Al}$ as the sacrificial layer

switching devices such as micromechanical relays [5]. Compared with polysilicon, TaSiN exhibits similar mechanical properties, the same or a longer lifetime and better electrical conductivity, which satisfies the requirements for a contact material.

\section{References}

[1] E. Kolawa, J.M. Molarius, C.W. Nieh and M.-A. Nicolet: Amorphous TaSiN thin-film alloys as diffusion barrier in Al/Si metallizations. J. Vac. Sci. Technol., A8 (1990) 3006-3010

[2] Jason S. Reid: Amorphous Ternary Diffusion Barriers for Silicon Matallizations. PhD Dissert-ation, California Institute of Technology (1995), Ch.2

[3] R.A. Buser and A. Dommann: Accurate microscopic method to investigate the aging of mocromachined silicon actuators. Sensors and actuators A, 43 (1994) 317-321

[4] G.F. McLane, L. Casas, J.S. Reid, E. Kolawa and M.-A. Nicolet: J. Vac. Sci. Technol., B12 (1994) 23522355

[5] M.-A. Grétillat, P. Thiébaud, N.F. de Rooji and C. Linder: Electrostatic polysilicon microrelays integrated with MOSFETs. IEEE Micro Electro Mech. Syst. Workshop, Oiso, Japan, (1994) 97-101
Elettra is a third generation synchrotron light source operated by Sincrotrone Trieste S.C.p.A and now engaged in user operation. The extremely high quality of the machine and beam lines has set new performance records in its field of operation and is regularly producing results of great scientific interest. The environment is multi-national and multi-disciplinary. Applications are invited from experienced researches for the position of beam line scientist at the SuperESCA beamline.

A PhD in physics, chemistry or materials science is required. The ideal candidate is a 30-35 year old researcher experienced with synchrotron radiation excited photoemission experiments and other surface science techniques. Capability of working as part of a team, working knowledge of spoken and written English, ability to understand users' needs and to communicate with them, extensive experience of Ultra High Vacuum instrumentation are required. A working knowledge of Italian would be preferable.

The duties include: development of advanced synchrotron radiation instrumentation. assistance of the ELETTRA users, with opportunities for independent or co-operative research.

The salary will be commensurate to the applicant's qualifications. Assistance with relocation costs within Europe will be offered. Applications including full curriculum vitae and the names and (electronic mail) addresses, telephone and fax numbers of three references should reach:

Sinchrotrone Trieste, S.S. $14 \mathrm{Km} 163.5$

in Area Science Park, 34012 Basovizza, TRIESTE

\section{Tutorial Workshop on \\ Quantum Information}

\section{Cryptography, Communication, and Computation \\ 17-19 October 1997, Almagro, Spain}

- The aim of the workshop is to provide introductory lectures on theoretical and experimental aspects of quantum information.

- Scientific Organization: TMR-network ..The Physics of Quantum Information", sponsored by the European Commission

- Organizing Committee: D. Bouwmeester, I. Cirac, A. Ekert, J.M. Riveiro, A. Zeilinger

- Local Organisation: University of Castilla-La-Mancha

- The deadline for registration is 1 August 1997. Registration and further information via email: Quantum-Information@uibk.ac.at. 